\title{
Search for signals of New Physics in Extensive Air Showers induced by Ultra High Energy Cosmic-Rays.
}

\author{
Juan de Dios Álvarez \\ Universidad Michoacana de San Nicolás de Hidalgo \\ E-mail: juandedios@ifm.umich.mx

\section{Umberto Cotti} \\ Universidad Michoacana de San Nicolás de Hidalgo \\ E-mail: ucottidifm.umich.mx
}

\section{Cederik de León*}

Universidad Autónoma de Puebla - Universidad Michoacana de San Nicolas de Hidalgo.

E-mail: cederikegmail.com

\begin{abstract}
The use of Cosmic Rays (CR) as a benchmark for new physics (beyond the Standard Model of particle physics) search, is an approach based on the fact that historically, the energy reached by the particle colliders has been and continues to be smaller than the energy the CR can achieve. Nevertheless the energies achieved by the particle colliders are getting closer to those of the CR of the highest energies, as a consequence the possible effects of new physics is now seeking mainly in CR of ultra high energy (UHECR), with an extremely low flux, resulting in a poor statistic but remains an opportunity to research for new physics.

In this work we present a general, model independent, analysis of the behavior of Ultra-High Energy Extensive Air Showers (UHE EAS) when some new effect, represented through the parametrization of a set of observables, are present and we determine on which range of this parameters could be possible to discern these kind of physics.
\end{abstract}

35th International Cosmic Ray Conference - ICRC2017

10-20 July, 2017

Bexco, Busan, Korea

${ }^{*}$ Speaker. 


\section{Introduction}

The search of new physics is based on validating the way that a model or theory produce effects with a defined, different from the Standard Model of particle physics (SM) and also detectable phenomenology. Searches for exotic new phenomena beyond the SM (BSM) are actually running at the LHC [1], as well as in non collider experiments [2] and particularly in UHECR [3]. The challenge in this approach, when used with UHECR, lies in the complexity of the parametrization of the phenomenology of a particular model and in the definition of the interactions of ultra high energy particles in the codes that simulates the development of the Extensive Air Shower (EAS).

In this work we separate the research from a particular model, to do this we determine a set of parameters that characterize the event and we vary this parameters to find the regions such that the detectable observables can indicate a behavior sufficiently distinct than the signal produced by a standard event.

The considered variables are associated to the production of massive particles capable to transport a certain amount (percentage) of the primary's energy as well as its capability to be enough long-lived to interact with the atmosphere before they decay and contribute, in that way, to the development of a part of the EAS.

We define an exotic EAS as that in which an exotic particle has been produced, considered as a particle able to transport an amount (percentage) of the energy of the primary enough to alter the development of the shower and generate a discernible signal from the standard EAS with the same primary characteristics but without exotic particles. Comparing some observables, between the exotic and the standard EAS, we determine for which values of the parameters that characterize the exotic EAS it is possible to distinguish it from the standard one.

We consider an energy range that start from one higher than the highest achieveble from LHC of $14 \mathrm{TeV}$ at center-of-mass $\sqrt{s}$. For an EAS generated by a proton with energy E that collides with a nucleon of the atmosphere of mass $m_{N}$, the value of $\sqrt{s}$ is approximately $\sqrt{2 m_{N} E}$. The detector with a unique quantity of data in this range is the Pierre Auger Observatory [4] which detected in a decade from 2004 to 2014 an amount of the order of $10^{5}$ events in an energy range from $\sim 10^{18}$ $\mathrm{eV}$ to $\sim 10^{20} \mathrm{eV}[5]$.

\section{Parametrization of the exotic Extensive Air Shower}

The EAS is produced as a consequence of the interaction of a primary cosmic ray with the atmosphere. This event is characterized by the CR type, its energy (E), its incident zenith angle $(\theta)$, its first interaction height a.s.l. $\left(h_{p}\right)$ (or its atmospheric depth). The variation of this parameters must be considered in a full analysis [6], in this first stage, we focus on the case of a vertical $(\theta=0)$ proton, with an energy of $E=10 \mathrm{EeV}$ and $h_{p}=25 \mathrm{~km}$.

A parametrization proposal for different kinds of exotic EAS is summarized in Figure [1] where the geometry of the kinematics of the events is defined. Six different combinations are presented, all with the same zenith angle $(\theta \neq 0)$, with different combinations of the following parameters: $h_{p}$ is the height a.s.l. where the first interaction of the primary proton occurs, $h_{e}$ is the height a.s.l. where the EAS associated to the exotic particle starts its development, $\beta$ is the angle between the EAS axis associated with the exotic particle and the EAS axis produced by the primary 
proton, $\alpha$ is the angle between the trajectory of the exotic, before the beginning of the associated shower, and the EAS axis produced by the primary proton. During the event that describes the first (or one of the firsts) interaction(s) a very massive exotic particle (or eventually a very heavy hadron) is generated and propagates with a determined trajectory carrying with it a fraction of the energy from the original primary proton.

According to the characteristics of the exotic particle, different scenarios can be presented that will be related to its critic energy, that in which its decay length is equal to its interaction length in the medium where it propagates. Depending on the critic energy of the exotic, a variety of exotic $E A S$ are possible to be developed as a function of its lifetime, short or long enough (short or long lived).

\subsection{Parameter space analysis, observables selection, methodology of the EAS generation and results.}

In relation with the primary's energy and its azimuth incident angle $(\theta)$, as we mentioned, it is considered for this first study only the case of a vertical proton with an energy of $10 \mathrm{EeV}$. For the atmospheric depth of the first interaction this is fixed in order to restrict, in the analysis of the libraries of the generated events, the presence of fluctuations, statistical and physical as well, associated to this parameter. A height of $25 \mathrm{~km}$ a.s.l. results to be suitable, being an approximate average of the first interactions of a proton of that energy $\left(h_{p}=25 \mathrm{~km}\right)$.

The energy fractions carried out by the exotic particle are analyzed in a range of $10 \%$ to $30 \%$ of the total energy of the primary, this range is scanned with increments of $10 \%$. The value for the height a.s.l. where the EAS associated to the exotic particle production begins to be manifest $\left(h_{e}\right)$ is varied between that which coincides with the primary proton $\left(h_{e}=h_{p}=25 \mathrm{~km}\right)$ until a limit located $5 \mathrm{~km}$ closer to the sea level, $5 \mathrm{~km}$ downwards, $\left(h_{e}=20 \mathrm{~km}\right)$, and that interval is scanned by increments of approximate $1500 \mathrm{~m}$. This combination of parameters correspond to the case IV of Figure [1].

The chosen observable to analyze the produced exotic EAS is the lateral profile from the particle density produced at ground level. The observables related with the longitudinal profile development have not generated, until this part of the analysis, evidences that allow to discern between a standard from an exotic EAS.

Considering an UHE-EAS ground detector, as the Pierre Auger Observatory, this analysis is realized focussing on the lateral profile for the muons density. The amount of generated EAS and the thinning used were different in order to optimize the CPU time in a balance between the number of necessary EAS vs. thinning [7].

The generation of the contribution to the EAS produced by the exotic event is implemented through the programming of a module within Aires [8]. The generation of the exotic contribution to the shower can be associated with either the (in)elastic interaction of the latter with the atmosphere or the interaction of the products of its decay. After performing several tests with different types of charged hadrons and having validated a common pattern of production of discernable results, the larger study was performed using a proton with the different energy levels already mentioned, which correspond to the type IV of the Figure [1].

As an example of the multiple generated events, we show in Figure [2] the lateral profiles of the muon density produced by three types of exotic events compared with a standard one. The three 

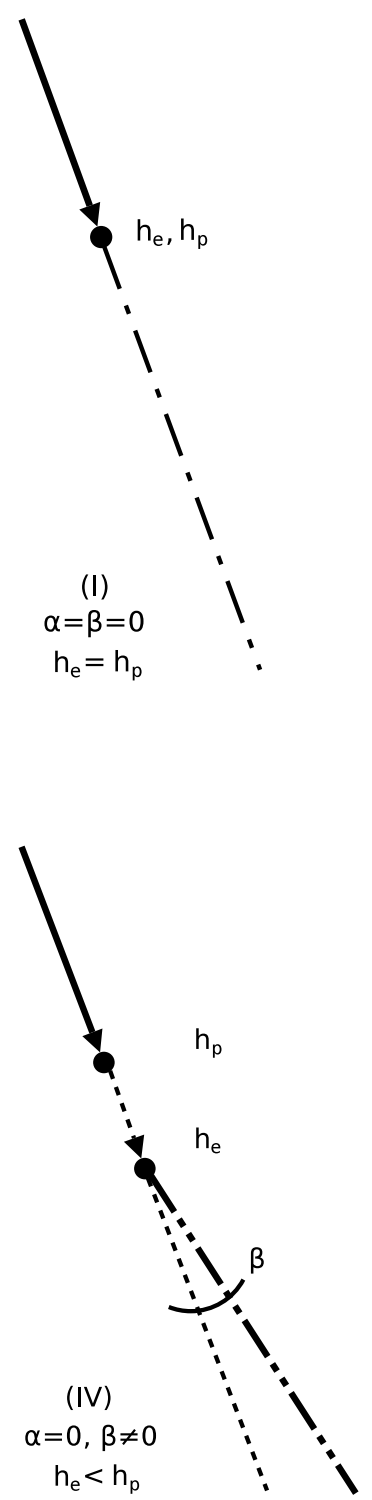
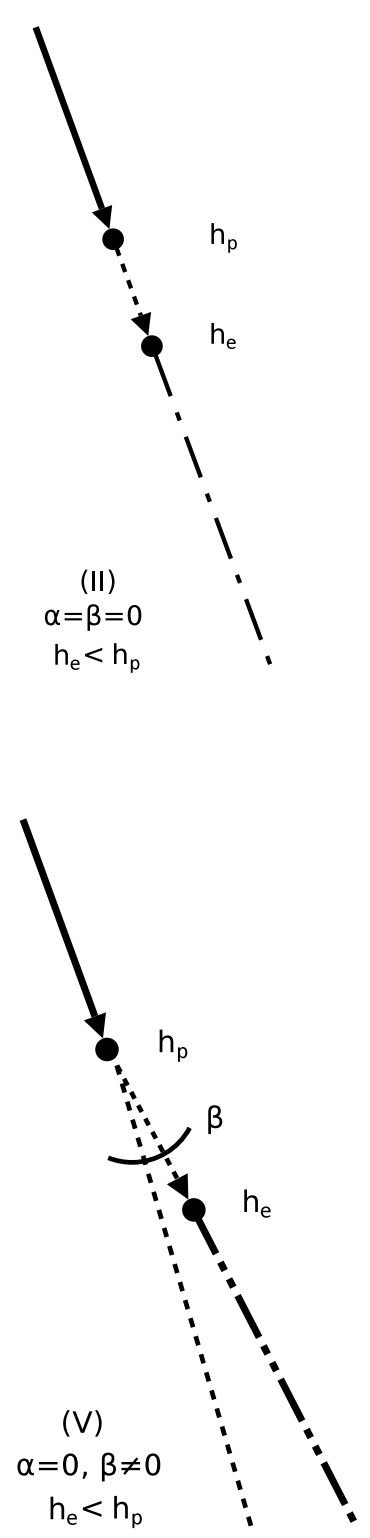
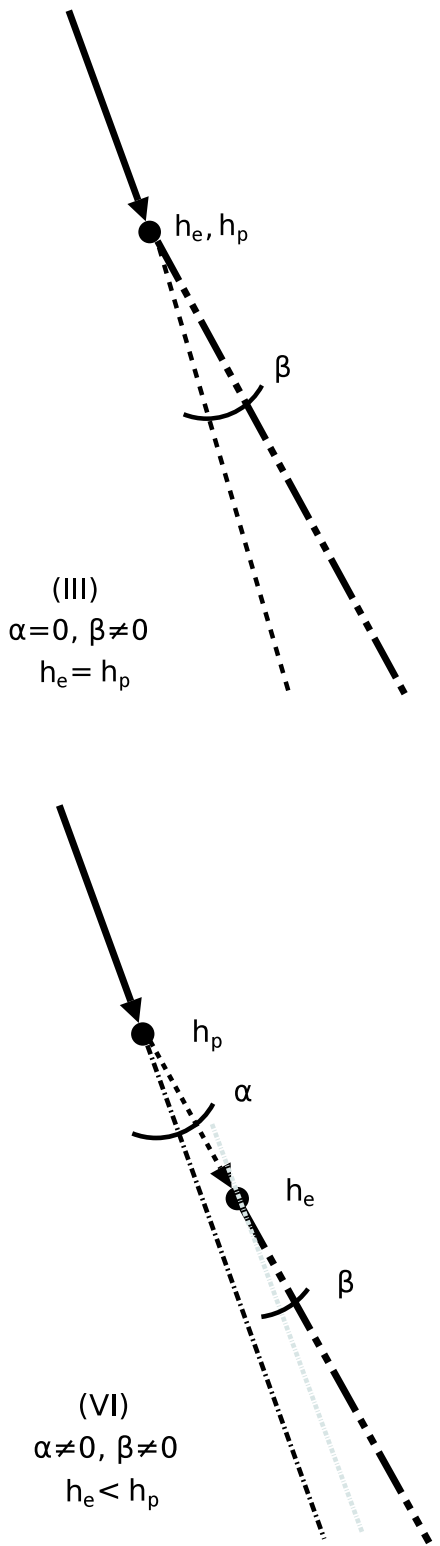

Figure 1: Different kinematics scenarios of the exotic EAS. In this case for proton with $\theta \neq 0, h_{p}$ is the height a.s.l. where the event related to the first interaction of the primary proton occurs, $h_{e}$ is the height a.s.l. where the event related to the first interaction of the exotic contribution occurs, $\beta$ as the angle between the EAS axis associated with the exotic particle and the EAS axis produced by the primary proton, $\alpha$ is the angle between the trajectory of the exotic, before of the beginning of the shower associated, and the EAS axis produced by the primary proton

exotics are associated to a value of $h_{e}=24 \mathrm{~km}$, all of them with the same energy, carrying $10 \%$ of the primary (originally $10 \mathrm{EeV}$ ) but with different production angles $(\beta)$, that is with the EAS axis associated to the exotic process forming angles of $0.5,1.0,1.5$ degrees with respect to the axis associated to the original proton. It can be observed that starting from angles $\sim 1.0$ degree there is an evident difference of the lateral profile of the muon density between the standard EAS and the 
exotic EAS.

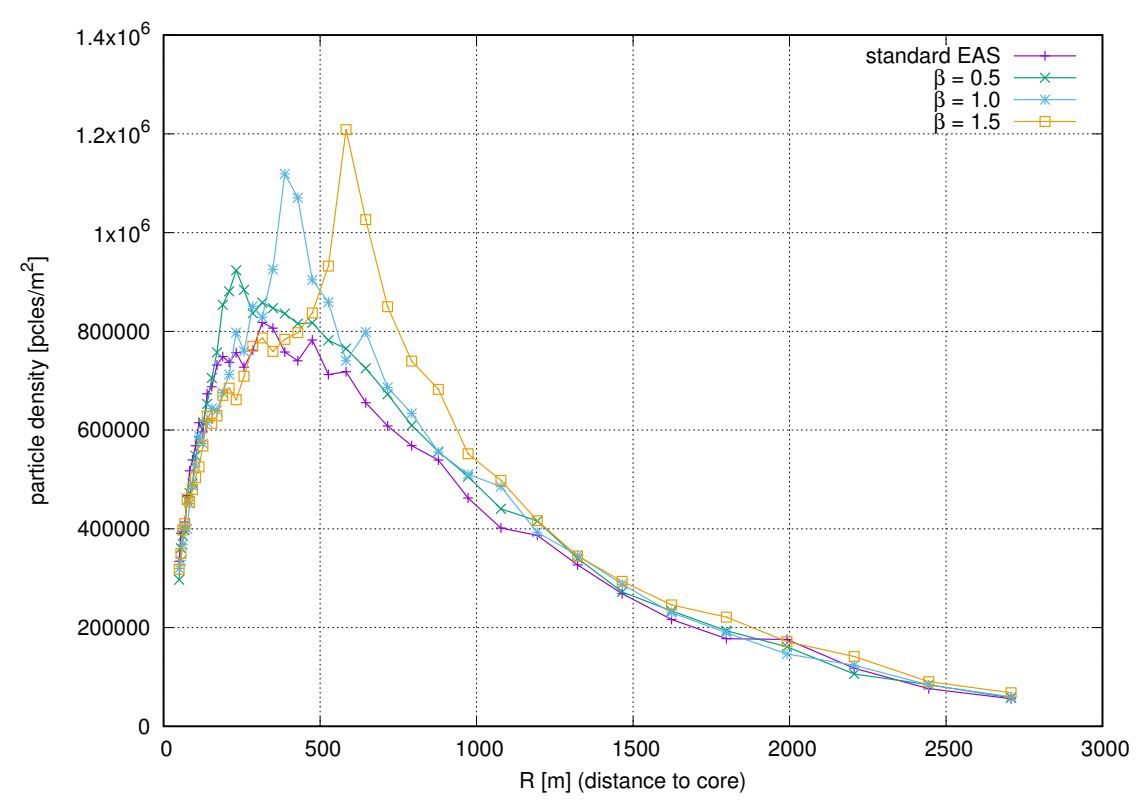

Figure 2: Muon lateral density profile related to three different exotic EAS compared with one standard EAS all with the same primary energy, initiated by a proton (primary particle), with energy $\mathrm{E}=10 \mathrm{EeV}$, incident zenith angle $\theta=0$, energy fraction for the exotic particle $10 \%$ (taken from primary), $h_{p}=25 \mathrm{~km}$ a.s.l. (altitude of the primary first interaction), $h_{e}=24 \mathrm{~km}$ a.s.l. (altitude of the first interaction of the section associated to the exotic particle contribution), and three different values of $\beta$ (exit angle of the exotic particle with respect to the axis shower core initiated by the primary particle).

Figure [3] represents the summary of the analysis for the whole set of parameters space studied. Every line is associated to exotic events that shares the same percentage of energy from the primary, while along the line the distance $h_{e}$ is varying, which characterize the first interaction for the exotic particle as a function of the angle $(\beta)$ subtended between the EAS axis associated with the exotic event and the original EAS. This line divide the parameter space $\left(\beta, h_{p}-h_{e}\right)$ in two regions, being the region to the right of every line, that in which the generation of an EAS associated to an exotic event can be manifested through the lateral profile of the muons density with characteristics clearly different from a standard one.

As an example, from Figure [3] one can determine that the production of an exotic particle generated with $10 \%$ of the primary's energy in a region closer to the first interaction(s) of the primary, could be detectable for values of $\beta$ of the order of one degree. Increasing the energy of the exotic, then the minimum values of detectability for $\beta$ will decrease up to values of $\beta=0.5$ degrees for percentages of the order of $30 \%$ of $\mathrm{E}$. 


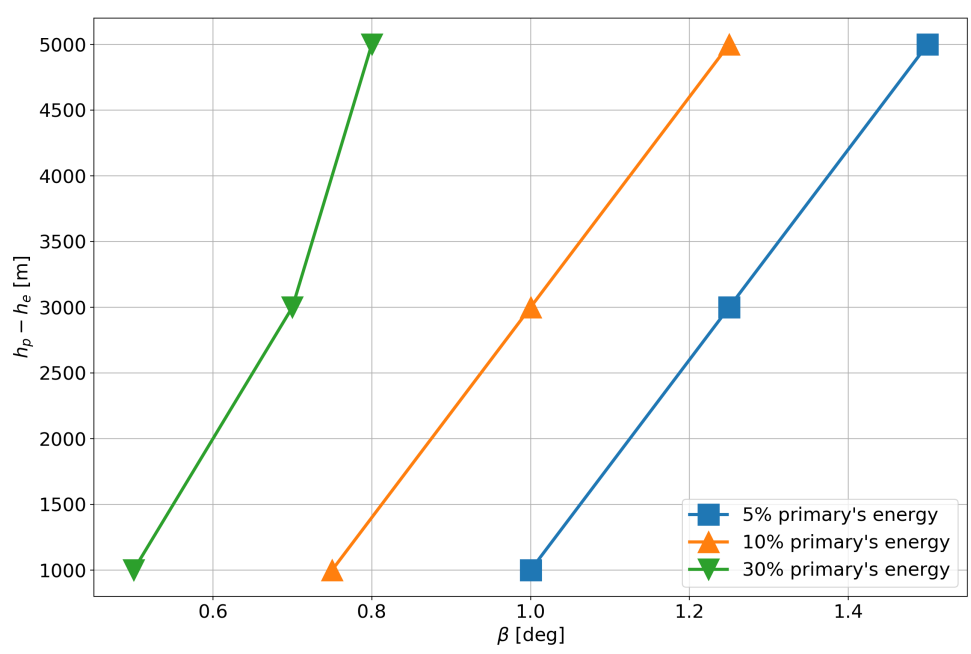

Figure 3: Summary of the performed analysis presented in the $\left(\beta, h_{p}-h_{e}\right)$ parameter space for an incident proton with a zenith angle $\theta=0$, an energy $\mathrm{E}=10 \mathrm{EeV}$ and with three different percentages of energy carried out by the exotic particle: 5\%,10\% and 30\%. Each line divide the parameter space in two regions, being the region to the right of every line, that in which the generation of an EAS associated to an exotic event can be manifested through the lateral profile of the muons density with characteristics clearly different from a standard one.

\section{Conclusions}

This work demonstrates that through the study of the lateral profile of the density distribution of an EAS, it is possible to observe the effect produced by an exotic particle that carries a relatively small fraction of the energy from the primary, and has a deviation of a few tenths of degree with respect to the primary shower axis.

This study has been focused on the analysis of the lateral shower profile, performing variation in the parameters previously mentioned. It has been found a minimum parameter set, and its values, under which could be observed an effect produced by the presence of an exotic particle created during the EAS development.

The results show that starting from a fraction of the $30 \%$ from the total energy of the primary and with a deviation as small as 0.4 degrees with respect to the primary shower axis, it is possible to observe a lateral profile with a clear evidence of the presence of a heavy particle into the EAS development.

The focus of this work is to keep it unlinked from the analysis of any particular model to determine, in this first approach, with which characteristics should met a model in order to produce detectable events.

It is considered it could be useful in the UHECR observatories in the process of reconstructions to eventually reconsider events that have been discarded for not being consistent with EAS generated in a standard physics context. 
Another field of implementation could be in the standard heavy hadrons generations $[9,10]$; with a particularly emphasis on the production of very low probability events.

\section{Acknowledgements}

The authors thankfully acknowledge the computer resources, technical expertise and support provided by the Laboratorio Nacional de Supercómputo del Sureste de México, CONACYT Network of national laboratories; To the Red de Física de Áltas Energías (Red FAE); VIEP-BUAP.

\section{References}

[1] T. Golling., LHC searches for exotic new particles, Prog.Part.Nucl.Phys. 2016 (90) 156

[2] Burdin S., Fairbairn M., Mermodc P., Milstead D., Pinfold J., Sloan T., Taylor W., Non-collider searches for stable massive particles, Phys.Rep. 2015 (582) 1 [arXiv: 1410.1374 ]

[3] J. I. Illana, M. Masip, and D. Meloni., New physics from ultrahigh energy cosmic rays., Phys. Rev., D75:055002 2007 [arXiv:hep-ph/0611036]

[4] The Pierre Auger Collaboration., Properties and performance of the prototype instrument for the Pierre Auger Observatory, Nucl.Instrum.Meth.A 2004 (523) 50

[5] Valiño I., The flux of ultra-high energy cosmic rays after ten years of operation of the Pierre Auger Observatory, ICRC 2015

[6] Cotti U., De León C. work in progress

[7] Álvarez J.deD., Cotti U., de León C., Computer time optimization in Extensive Air Shower simulations, ICRC $\mathbf{2 0 1 7}$

[8] Sciutto S.J.. AIRES, a system for air shower simulation and analysis, Proc. 27th ICRC 2001 (1) 237

[9] García Canal C.A., Illana J.I., Masip M., Sciutto S.J., Production and propagation of heavy hadrons in air-shower simulators, Astropart.Phys. 2013 (46) 29

[10] Gonçalves V.P., Gratieri D.R., Heavy quark production in ultra high energy cosmic ray interactions, Astropart.Phys. 2015 (61) 46 [arXiv: 1402.0418 ] 